To be presented at the $32^{\text {nd }}$ AIAA/ASME/SAE/ASEE Joint Propulsion Conference,

Lake Buena Vista, FL, July 1-3, 1996

\section{A SURVEY OF COMBUSTIBLE METALS, THERMITES, AND INTERMETALLICS FOR PYROTECHNIC APPLICATIONS*}

\author{
S. H. Fischer and M. C. Grubelich \\ Sandia National Laboratories \\ Albuquerque, NM 87185-1453
}

\begin{abstract}
Thermite mixtures, intermetallic reactants, and metal fuels have long been used in pyrotechnic applications. Advantage of these systems typically include high energy density, impact insensitivity, high combustion temperature, and a wide range of gas production. They generally exhibit high temperature stability, and possess insensitive ignition properties. In this paper, we review the applications, benefits, and characteristics of thermite mixtures, intermetallic reactants, and metal fuels.
\end{abstract}

\section{INTRODUCTION}

Exothermic reactions between a metal and a metal oxide (thermite), between metallic elements (intermetallic), and the combustion of metals (metal oxidation reactions) are extremely useful sources of energy production and material synthesis for numerous applications. For example, the thermite welding process was first demonstrated in 1898 and continues to be the most frequently used method for the field welding of railroad track. ${ }^{1,2}$

Other applications for thermite reactions include: thermite torches for underwater and atmospheric cutting and perforation; electronic hardware destruct devices; additives to propellants and explosives for increased performance; pyrotechnic switches; airbag gas generator materials; reactive fragments; hightemperature-stable igniters; free-standing insertable heat sources; devices to breech ordnance cases to relieve pressure during fuel fires; and methods of producing alumina liners in situ for pipes.

Applications for intermetallic reactions include: consumable port covers for ramjet engine inlets; tracer compositions for munitions; ramjet fuels; self-ejecting combustible plumes for large-area heating; ignition aids for thermites; thermal battery heat sources; incendiary projectiles; delay fuzes; additives to propellants to increase burn rate without significant decrease of specific impulse; and shaped-charge liners.

* This work was supported by the United States Department of Energy under Contract DE-AC04-94AL85000.
Metal fuels have been used as: additives to increase shock sensitivity of explosives; additives to increase explosive blast effects; fuel-air explosives; additives to both solid and liquid propellants to increase density impulse; methods of controlling combustion instability in solid propellant rockets; additives to solid and liquid fuels for ramjets to increase range; and fuels in numerous pyrotechnic devices. ${ }^{2-22}$

Thermite, intermetallic, and metal fuels (with an JUL 22 经通 oxidizer) can be ignited via a thermal impulse from a hot-wire, exploding bridgewire (EBW), or semiconductor bridge (SCB) igniter as well as by laser impingement, mechanical methods, or shock initiation. ${ }^{12,23-29}$ Many of these formulations are stable at high temperatures and are insensitive to the effects of moisture, corrosion, friction, spark, shock, contaminants, and variations in composition ${ }^{15}$. Clearly, these types of exothermic reaction mixtures provide the output for a wide variety of engineering applications with a large choice of ignition methods.

\section{CALCULATIONS}

The "traditional" thermite reaction is taken as the reaction of a stoichiometric mix of aluminum and magnetite $\left(\mathrm{Fe}_{3} \mathrm{O}_{4}\right)$ reacting exothermically to completion to the products alumina $\left(\mathrm{Al}_{2} \mathrm{O}_{3}\right)$ and iron. Many other thermite mixtures exist; many of these, as well as intermetallic and metal-oxidation reactions, are surveyed in this paper. Some of these reactions produce little or no gas. Others produce significant amounts of gaseous products. The reactant composition can be chosen to produce solid, liquid, and/or gaseous products as required for the particular application. ${ }^{6,30,31}$

Table 1 lists the theoretical maximum density (TMD) of the reactants, the adiabatic reaction temperature with and without taking into account the heats of phase changes, the state of the products, the amount of gas produced referenced to the total mass of the reactants (or products), and the heat of reaction based on the mass and volume of the reactants for a selection of exothermic thermite reactions. The same information for intermetallic reactions is listed in Table 2. In the field of intermetallic reactions, boron, carbon, and silicon are usually considered metallic. ${ }^{15}$ In the present study, sulfur was also included. Analogous values (metal density and heat of reaction with respect to the mass and volume of the metal) for metal-oxidation 
reactions are listed in Table 3. Physical, thermochemical, and reaction data were taken from references $13,14,17,18,28$, and 32-47.

The heat of reaction was calculated assuming complete adiabatic reaction of the reactants starting at $298 \mathrm{~K}$. The increase in temperature was calculated using the average specific heat over the temperature range from $298 \mathrm{~K}$ to the adiabatic reaction temperature. If phase transitions (solid-solid, solid-liquid, or liquid-gas) occurred over that temperature range, the adiabatic reaction temperature was calculated taking into account the heats of those transitions and using the average specific heats for each temperature range between transitions. This calculated temperature is an upper limit for the ideal case of complete combustion and no energy losses.

In most of the calculations reported in the open literature, the adiabatic reaction temperature is calculated by the first method without taking into account the heats of the phase transitions. This does a inadequate job of dealing with the changes in heat capacity as phase changes occur and leads to erroneously high temperatures. For instance, for "traditional" thermite $\left(8 \mathrm{Al}+3 \mathrm{Fe}_{3} \mathrm{O}_{4}\right)$ the adiabatic reaction temperature with no phase transitions taken into account was calculated as $4057 \mathrm{~K}$. In contrast, with the solid-solid, solid-liquid, and liquid-gas heats of transition included, the adiabatic reaction temperature was more accurately calculated as $3135 \mathrm{~K}$. Similarly, for $\mathrm{Ti}+2 \mathrm{~B}$, the calculated adiabatic reaction temperature dropped from $3710 \mathrm{~K}$ to $3498 \mathrm{~K}$. Measured reaction temperatures are in reasonable agreement with the calculated values. Temperatures ranging from $2800 \mathrm{~K}$ to $3000 \mathrm{~K}$ have been measured for $8 \mathrm{Al}+3 \mathrm{Fe}_{3} \mathrm{O}_{4}$, while that for $\mathrm{Ti}+2 \mathrm{~B}$ has been measured in the range of $3150 \mathrm{~K}$ to $3300 \mathrm{~K}$. $15,47,50$

An accurate calculation of the adiabatic reaction temperature is important for determining whether the reaction is likely to be self-propagating. A strong indication that the reaction is self-propagating is if at least one of the product species is brought to its melt temperature. ${ }^{43}$ Another indication that a reaction is self-propagating is an adiabatic reaction temperature greater than 2000K. ${ }^{17}$ (Reactions which are not selfpropagating under normal conditions may become so when initiated by a high-power stimulus, such as a high-energy shock. Self-propagation can also be promoted by preheating the reactants to a high temperature. ${ }^{43}$ ) It should be noted that, since the effect of phase changes on the product temperature takes a finite time, the initial temperature rise may control the diffusion and reaction rates before the temperature drops due to the phase changes. ${ }^{28}$

The reaction temperature is also a guide as to which materials are suitable for a given application. For some applications, such as cutting through metal, high temperatures are required. For others, such as air-bag inflation, low-temperature products are desirable.

\section{DISCUSSION}

For engineering applications, the "optimal" exothermic mixture is dependent on several factors which include: the energy per unit mass (or volume depending on the requirements of the application); the chemical stability of the reactants and products at normal operating temperatures; the chemical compatibility of the reactants and products with other materials present in the application; the toxicity of the reactants and products; the reaction rate; ease of processing; availability of the reactants; reaction temperature; state of the products; and cost.

From Tables 1 and 2, thermite and intermetallic compositions can be selected to produce solid, liquid, or gaseous reaction products as required for a particular application. In situations where gas production is undesirable, such as obturated systems, applications for which it is desirable to control the reaction rate by conduction rather than convection, or systems which may be adversely affected by pressure variations, solid and liquid products are more suitable. However, in order to perform mechanical work, rapidly convey the product (as in a torch-type output), or inflate items such as airbags, the production of gases is required.

For the selection of metal fuels similar compromises apply. Desirable properties for metal fuels are a high heat of combustion per unit mass of metal (or of the metal and the oxidizer for some applications), a high density, and low melt and vaporization temperatures. Table 3 contains several properties of importance. Ideally one would select boron or beryllium based on their high energy content. Unfortunately, low combustion efficiency and toxicity, respectively, limit the application of these metals. In general, the wide use of aluminum in propellant, pyrotechnic, and explosive formulations is because of its many desirable properties. Most other metals have applications in systems requiring very specific properties. For example, zirconium is used where ignition sensitivity and high reaction rates are required, while copper is used when a good heat conductor is necessary. 


\section{DISCLAIMER}

This report was prepared as an account of work sponsored by an agency of the United States Government. Neither the United States Government nor any agency thereof, nor any of their employees, makes any warranty, express or implied, or assumes any legal liability or responsibility for the accuracy, completeness, or usefulness of any information, apparatus, product, or process disclosed, or represents that its use would not infringe privately owned rights. Reference herein to any specific commercial product, process, or service by trade name, trademark, manufacturer, or otherwise does not necessarily constitute or imply its endorsement, recommendation, or favoring by the United States Government or any agency thereof. The views and opinions of authors expressed herein do not necessarily state or reflect those of the United States Government or any agency thereof. 


\section{DISCLAIMER}

Portions of this document may be illegible in electronic image products. Images are produced from the best available original document. 


\section{SUMMARY}

Numerous thermite and intermetallic energetic compositions exist that can be used for a wide variety of engineering applications. Metal combustion reactions are also of great utility. A comprehensive list of these materials and their energetic properties, including a computation of adiabatic reaction temperatures with and without phase changes, was presented here. Comparison to experimentally measured reaction temperatures shows reasonable agreement with the calculated adiabatic reaction temperatures. The heats of reaction and temperatures provide a useful guide for choosing exothermic formulations for engineering applications.

\section{REFERENCES}

1. E. Moin, The Current Status of Field Welding of Rail, Railway Track Struct., October 1988.

2. A. A. Shidlovskiy, Principles of Pyrotechnics, Mashinostroyeniye Press, 1964.

3. J. H. Mohler, D. L. Halcomb, and D. R. Begeal, $A n$ Effective Low-Profile Thermite Torch, MLM--3650(OP), 1990.

4. S. J. Marziano and R. E. Donnard, Thermite Penetrator Device, U. S. Patent 4,216,721, 1980.

5. S. Calsson and H. Schmid, Rocket and Ramjet Propellants, European Patent Application 0487 $473 \mathrm{Al}, 1991$.

6. J. C. Hinshaw and R. J. Blau, Thermite Compositions for Use as Gas Generators, International Application WO 95/04672, 1995.

7. A. Gibson, L. D. Haws, and J. H. Mohler, Integral Low-Energy Thermite Igniter, PATENTS-US--A6494487, 1983.

8. J. H. Westbrook, Miscellaneous Applications, Chapter 31 in Intermetallic Compounds, Vol. 2, Practice, ed. J. H. Westbrook and R. L. Fleisher, pp. 645-656, 1994.

9. A. C. Munger, J. H. Mohler, and M. D. Kelly, Feasibility of a Free-Standing Insertable Heat Source, Proc. Int. Pyrotechnics Seminar, $8^{\text {th }}, 496-$ $511,1982$.

10. M. A. Riley, P. D. Zavitsanos, C. Files, D. Walz, and A. C. Ratzel, Particulate Plume Radiation via Intermetallic Reactions, SAND91-2696A, abstract for presentation to Int. Pyro. Soc. Ann. Meeting, July 1992, Breckenridge, CO.

11. J. H. McLain, Pyrotechnics from the Viewpoint of Solid State Chemistry, Franklin Institute Press, 1980.

12. A. P. Hardt, Study of Tracer Munitions Using Intermetallic Reactions, FA-TR-74043, 1974.

13. R. J. Hancox, The Development of Plastic Thermite, MRL-R-868, 1983.
14. R. J. Hancox, The Development of Plastic Thermite, Proc. Int. Pyrotechnics Seminar, gth, 257-274, 1984.

15. A. P. Hardt, Incendiary Potential of Exothermic Intermetallic Reactions, AFATL-TR-71-87, 1971.

16. J. L. Prentice, Heat Sources for Thermal Batteries: Exothermic Intermetallic Reactions, US Patent 4,158,084, 1979.

17. L. L. Wang, Z. A. Munir, and Y. M. Maximov, "Thermite Reactions: Their Utilization in the Synthesis and Processing of Materials, J. Matls. Sci 28 (1993) 3693-3708, 1993.

18. C. S. Cross, Improvements in or relating to Explosive Compositions, Patent Specification 1,165,027, 1969.

19. J. Covino, Bonding Agents for Thermite Compositions, US Patent 5,035,756, 1989.

20. H. E. Montgomery, Jr., Reactive Fragment, U. S. Patent 3,961,576, 1976.

21. J. A. Trimble and T. D. Meyers, Nonejectable Port Cover for Ramjet Engines, CPIA-PUB-455-VOLIII, JANNAF Propulsion Meeting, 1986.

22. R. F. Vetter, Rocket Motor Thermal Case Penetrator - An Approach to Fast Cookoff Hazard Reduction, CPIA-PUB-425-VOL-III, JANNAF Propulsion Meeting, 1985.

23. N. Le Poidevin, A Fuse for Thermite Reaction, letter to Sch Science Review 48164 (243-4), 1966.

24. A. C. Munger and M. D. Kelly, Parameters Affecting Hot Wire Ignition of Thermite Mixtures, Proc. Int. Pyrotechnics Seminar, $9^{\text {th }}, 405-414$, 1984.

25. R. W. Bickes, Jr., M. C. Grubelich, S. M. Harris, J. A. Merson, J. H. Weinlein, An Overview of Semiconductor Bridge, SCB, Applications at Sandia National Laboratories," $31^{\text {st }}$ AIAA Joint Propulsion Conference and Exhibit, San Diego, July 10-12, 1995, SAND95-0968C.

26. A. P. Hardt, Shock Initiation of Thermite, Proc. Int. Pyrotechnic Seminar, 13 th $, 425-438,1988$.

27. L. L. Wang, Z. A. Munir, and J. B. Holt, J. Matls. Synthesis and Processing, vol. 2, No. 4, 1994.

28. P. V. Phung and A. P. Hardt, "Ignition Characteristics of Gasless Reactions," Comb. and Flame 22 323-335 (1974).

29. R. W. Bickes, Jr., M. C. Grubelich, J. A. Romero, D. J. Staley, R. J. Buss, P. P. Ward, and K. L. Erickson, $A$ New Concept for Very Low Energy Detonators and Torches, SAND96-0703, March 1996.

30. D. L. Halcomb and J. H. Mohler, High- and LowTemperature-Stable Thermite Composition for Producing High-Pressure, High-Velocity Gases, U. S. Patent 4,963,203, 1990. 
31. R. J. Hancox, Compositions and Devices for High Temperature Combustion, Patent Application WO 85/00364, EP0148252, 1985.

32. TAPP Thermochemical and Physical Properties, Version 2.2, E. S. Microware, 1994.

33. G. V. Samsanov, The Oxide Handbook, Inst. of Problems in Materials Science, Academy of Sciences of the Ukrainian SSR, Kiev, USSR.

34. I. Barin, Thermochemical Data of Pure Substances, 1989, VCH.

35. I. Barin, O Knacke, and O. Kubaschewski, Thermochemical Properties of Inorganic Substances, 1977, Springer-Verlag.

36. N. A. Lange, Handbook of Chemistry, Tenth Edition, 1961.

37. R. H. Perry and C. H. Chilton, Chemical Engineer's Handbook, $5^{\text {th }}$ Edition, 1973.

38. R. C. Weast and M. J. Astle, CRC Handbook of Chemistry and Physics, 59th Edition, CRC Press, 1978.

39. C. E. Wicks and F. E. Block, Thermodynamic Properties of 65 Elements-Their Oxides. Halides, Carbides, and Nitrides, Bureau of Mines, U. S. Dept. of Interior, 1963.

40. W. H. Gitzen, Alumina As a Ceramic Material, ACS, 1970.

41. Bismuth and Bismuth Alloys, in Kirk-Othmer Encyclopedia of Chemical Technology, Volume 4, John Wiley \& Sons, 1992

42. A. A. Shidlovskii and V. V. Gorbunov, "Combustion of Nickel-Aluminum Thermite," Fizika Goreniya I Vzryva, vol. 18, no. 4, 420-422, 1983.

43. A. P. Hardt and P. V. Phung, "Propagation of Gasless Reactions in Solids - I. Analytical Study of Exothermic Intermetallic Reactions Rates." Comb. And Flame 21 77-89, 1973.

44. J. Mohler, personal communications, 1996.

45. Y. S. Touloukian, Thermophysical Properties of High Temperature Solid Materials, 1967.

46. J. V. Goodfellow, Improvements in or relating to Explosive Compositions, Patent Spec. 1,165,027, 1969.

47. A. G. Merzhanov, Pyrotechnical Aspects of SelfPropagating High-Temperature Synthesis (SHS), NSWCCR/RDTN-94/004, 1994.

48. V. A. Begolyubov, Temperature of an Aluminothermic Process as a Function of Heat Evolved per Kilogram, Stal' 17, 531-535, 1957 (translation SAND80-6013).

49. D. A. Powers and F. E. Arellano, Direct Observation of Melt Behavior During High Temperature Melt/Concrete Interaction, SAND81-1754, 1982.
50. W. W. Tarbell, R. E. Blose, and F. E. Arellano, Molten Thermite Teeming Into an Iron Oxide Particle Bed, SAND82-2475, 1984. 
Table 1 - Thermite Reactions

\begin{tabular}{|c|c|c|c|c|c|c|c|c|c|}
\hline \multicolumn{2}{|c|}{ reactants } & \multicolumn{2}{|c|}{$\begin{array}{c}\text { adiabatic reaction } \\
\text { temperature }(\mathbf{K})\end{array}$} & \multicolumn{2}{|c|}{ state of products } & \multicolumn{2}{|c|}{ gas production } & \multicolumn{2}{|c|}{ heat of reaction } \\
\hline constituents & $\begin{array}{l}\rho_{\mathrm{TMD}} \\
\mathrm{g} / \mathrm{cm}^{3}\end{array}$ & $\begin{array}{l}\text { w/o phase } \\
\text { changes }\end{array}$ & $\begin{array}{l}\text { w/ phase } \\
\text { changes }\end{array}$ & $\begin{array}{l}\text { state of } \\
\text { oxide }\end{array}$ & $\begin{array}{l}\text { state of } \\
\text { metal }\end{array}$ & $\begin{array}{l}\text { moles gas } \\
\text { per } 100 \mathrm{~g}\end{array}$ & $\begin{array}{l}\mathrm{g} \text { of gas } \\
\text { per } \mathrm{g}\end{array}$ & $\begin{array}{l}-Q, \\
\mathrm{cal} / \mathrm{g}\end{array}$ & $\begin{array}{c}-Q, \\
\mathrm{cal} / \mathrm{cm}^{3}\end{array}$ \\
\hline $2 \mathrm{Al}+3 \mathrm{AgO}$ & 6.085 & 7503 & 3253 & l-g & gas & 0.7519 & 0.8083 & 896.7 & 5457 \\
\hline $2 \mathrm{Al}+3 \mathrm{Ag}_{2} \mathrm{O}$ & 6.386 & 4941 & 2436 & liquid & l-g & 0.4298 & 0.4636 & 504.8 & 3224 \\
\hline $2 \mathrm{Al}+\mathrm{B}_{2} \mathrm{O}_{3}$ & 2.524 & 2621 & 2327 & $\mathrm{~s}-1$ & solid & 0.0000 & 0.0000 & 780.7 & 1971 \\
\hline $2 \mathrm{Al}+\mathrm{Bi}_{2} \mathrm{O}_{3}$ & 7.188 & 3995 & 3253 & $\log$ & gas & 0.4731 & 0.8941 & 506.1 & 3638 \\
\hline $2 \mathrm{Al}+3 \mathrm{CoO}$ & 5.077 & 3392 & 3201 & liquid & $1-g$ & 0.0430 & 0.0254 & 824.7 & 4187 \\
\hline $8 \mathrm{Al}+3 \mathrm{Co}_{3} \mathrm{O}_{4}$ & 4.716 & 3938 & 3201 & liquid & $1-g$ & 0.2196 & 0.1294 & 1012 & 4772 \\
\hline $2 \mathrm{Al}+\mathrm{Cr}_{2} \mathrm{O}_{3}$ & 4.190 & 2789 & 2327 & $s-1$ & liquid & 0.0000 & 0.0000 & 622.0 & 2606 \\
\hline $2 \mathrm{Al}+3 \mathrm{CuO}$ & 5.109 & 5718 & 2843 & liquid & l-g & 0.5400 & 0.3431 & 974.1 & 4976 \\
\hline $2 \mathrm{Al}+3 \mathrm{Cu}_{2} \mathrm{O}$ & 5.280 & 4132 & 2843 & liquid & $1-g$ & 0.1221 & 0.0776 & 575.5 & 3039 \\
\hline $2 \mathrm{Al}+\mathrm{Fe}_{2} \mathrm{O}_{3}$ & 4.175 & 4382 & 3135 & liquid & l-g & 0.1404 & 0.0784 & 945.4 & 3947 \\
\hline $8 \mathrm{AI}+3 \mathrm{Fe}_{3} \mathrm{O}_{4}$ & 4.264 & 4057 & 3135 & liquid & $\mathrm{l}-\mathrm{g}$ & 0.0549 & 0.0307 & 878.8 & 3747 \\
\hline $2 \mathrm{Al}+3 \mathrm{HgO}$ & 8.986 & 7169 & 3253 & $\mathrm{l}-\mathrm{g}$ & gas & 0.5598 & 0.9913 & 476.6 & 4282 \\
\hline $10 \mathrm{Al}+3 \mathrm{I}_{2} \mathrm{O}_{5}$ & 4.119 & 8680 & $>3253^{*}$ & gas & gas & 0.6293 & 1.0000 & 1486 & 6122 \\
\hline $4 \mathrm{Al}+3 \mathrm{MnO}_{2}$ & 4.014 & 4829 & 2918 & liquid & gas & 0.8136 & 0.4470 & 1159 & 4651 \\
\hline $2 \mathrm{Al}+\mathrm{MoO}_{3}$ & 3.808 & 5574 & 3253 & $\mathrm{l}-\mathrm{g}$ & liquid & 0.2425 & 0.2473 & 1124 & 4279 \\
\hline $10 \mathrm{Al}+3 \mathrm{Nb}_{2} \mathrm{O}_{5}$ & 4.089 & 3240 & 2705 & liquid & solid & 0.0000 & 0.0000 & 600.2 & 2454 \\
\hline $2 \mathrm{Al}+3 \mathrm{NiO}$ & 5.214 & 3968 & 3187 & liquid & $\mathrm{l}-\mathrm{g}$ & 0.0108 & 0.0063 & 822.3 & 4288 \\
\hline $2 \mathrm{Al}+\mathrm{Ni}_{2} \mathrm{O}_{3}$ & 4.045 & 5031 & 3187 & liquid & $1-g$ & 0.4650 & 0.2729 & 1292 & 5229 \\
\hline $2 \mathrm{Al}+3 \mathrm{PbO}$ & 8.018 & 3968 & 2327 & s-1 & gas & 0.4146 & 0.8591 & 337.4 & 2705 \\
\hline $4 \mathrm{Al}+3 \mathrm{PbO}_{2}$ & 7.085 & 6937 & 3253 & $\mathrm{l}-\mathrm{g}$ & gas & 0.5366 & 0.9296 & 731.9 & 5185 \\
\hline $8 \mathrm{Al}+3 \mathrm{~Pb}_{3} \mathrm{O}_{4}$ & 7.428 & 5427 & 3253 & l-g & gas & 0.4215 & 0.8466 & 478.1 . & 3551 \\
\hline $2 \mathrm{Al}+3 \mathrm{PdO}$ & 7.281 & 5022 & 3237 & liquid & $\log$ & 0.6577 & 0.6998 & 754.3 & 5493 \\
\hline $4 \mathrm{Al}+3 \mathrm{SiO}_{2}$ & 2.668 & 2010 & 1889 & solid & liquid & 0.0000 & 0.0000 & 513.3 & 1370 \\
\hline $2 \mathrm{Al}+3 \mathrm{SnO}$ & 5.540 & 3558 & 2876 & liquid & $1-g$ & 0.1070 & 0.1270 & 427.0 & 2366 \\
\hline $4 \mathrm{Al}+3 \mathrm{SnO}_{2}$ & 5.356 & 5019 & 2876 & liquid & l-g & 0.2928 & 0.3476 & 686.8 & 3678 \\
\hline $10 \mathrm{Al}+3 \mathrm{Ta}_{2} \mathrm{O}_{5}$ & 6.339 & 3055 & 2452 & liquid & solid & 0.0000 & 0.0000 & 335.6 & 2128 \\
\hline $4 \mathrm{Al}+3 \mathrm{TiO}_{2}$ & 3.590 & 1955 & 1752 & solid & liquid & 0.0000 & 0.0000 & 365.1 & 1311 \\
\hline $16 \mathrm{Al}+3 \mathrm{U}_{3} \mathrm{O}_{8}$ & 4.957 & 1406 & 1406 & solid & solid & 0.0000 & 0.0000 & 487.6 & 2417 \\
\hline $10 \mathrm{Al}+3 \mathrm{~V}_{2} \mathrm{O}_{5}$ & 3.107 & 3953 & 3273 & $1-\mathrm{g}$ & liquid & 0.0699 & 0.0356 & 1092 & 3394 \\
\hline $4 \mathrm{Al}+3 \mathrm{WO}_{2}$ & 8.085 & 4176 & 3253 & l-g & solid & 0.0662 & 0.0675 & 500.6 & 4047 \\
\hline $2 \mathrm{AI}+\mathrm{WO}_{3}$ & 5.458 & 5544 & 3253 & $1-g$ & liquid & 0.1434 & 0.1463 & 696.4 & 3801 \\
\hline $2 \mathrm{~B}+\mathrm{Cr}_{2} \mathrm{O}_{3}$ & 4.590 & 977 & 917 & liquid & solid & 0.0000 & 0.0000 & 182.0 & 835.3 \\
\hline $2 \mathrm{~B}+3 \mathrm{CuO}$ & 5.665 & 4748 & 2843 & gas & $\log$ & 0.4463 & 0.2430 & 738.1 & 4182 \\
\hline $2 \mathrm{~B}+\mathrm{Fe}_{2} \mathrm{O}_{3}$ & 4.661 & 2646 & 2065 & liquid & liquid & 0.0000 & 0.0000 & 590.1 & 2751 \\
\hline $8 \mathrm{~B}+3 \mathrm{Fe}_{3} \mathrm{O}_{4}$ & 4.644 & 2338 & 1903 & liquid & liquid & 0.0000 & 0.0000 & 530.1 & 2462 \\
\hline $4 \mathrm{~B}+3 \mathrm{MnO}_{2}$ & 4.394 & 3000 & 2133 & $\mathrm{l}-\mathrm{g}$ & liquid & 0.3198 & 0.1715 & 773.1 & 3397 \\
\hline $8 \mathrm{~B}+3 \mathrm{~Pb}_{3} \mathrm{O}_{4}$ & 8.223 & 4217 & 2019 & liquid & l-g & 0.4126 & 0.8550 & 326.9 & 2688 \\
\hline $3 \mathrm{Be}+\mathrm{B}_{2} \mathrm{O}_{3}$ & 1.850 & 3278 & 2573 & liquid & $s-1$ & 0.0000 & 0.0000 & 1639 & 3033 \\
\hline $3 \mathrm{Be}+\mathrm{Cr}_{2} \mathrm{O}_{3}$ & 4.089 & 3107 & 2820 & $s-1$ & liquid & 0.0000 & 0.0000 & 915.0 & 3741 \\
\hline
\end{tabular}

- More data needed for this calculation. 
Table 1 - Thermite Reactions (cont.)

\begin{tabular}{|c|c|c|c|c|c|c|c|c|c|}
\hline \multicolumn{2}{|c|}{ reactants } & \multicolumn{2}{|c|}{$\begin{array}{c}\text { adiabatic reaction } \\
\text { temperature }(K)\end{array}$} & \multicolumn{2}{|c|}{ state of products } & \multicolumn{2}{|c|}{ gas production } & \multicolumn{2}{|c|}{ heat of reaction } \\
\hline constituents & $\begin{array}{l}\rho_{\mathrm{TMm}}, \\
\mathrm{g} / \mathrm{cm}^{3}\end{array}$ & $\begin{array}{l}\text { w/o phase } \\
\text { changes }\end{array}$ & $\begin{array}{l}\text { w/ phase } \\
\text { changes }\end{array}$ & $\begin{array}{l}\text { state of } \\
\text { oxide }\end{array}$ & $\begin{array}{c}\text { state of } \\
\text { metal }\end{array}$ & $\begin{array}{l}\text { moles gas } \\
\text { per } 100 \mathrm{~g}\end{array}$ & $\begin{array}{c}\mathrm{g} \text { of gas } \\
\text { per } \mathrm{g}\end{array}$ & $\begin{array}{l}-Q \\
\mathrm{cal} / \mathrm{g}\end{array}$ & $\begin{array}{c}-Q, \\
\mathrm{cal} / \mathrm{cm}^{3}\end{array}$ \\
\hline $\mathrm{Be}+\mathrm{CuO}$ & 5.119 & 3761 & 2820 & $\mathrm{~s}-1$ & liquid & 0.0000 & 0.0000 & 1221 & 6249 \\
\hline $3 \mathrm{Be}+\mathrm{Fe}_{2} \mathrm{O}_{3}$ & 4.163 & 4244 & 3135 & liquid & l-g & 0.1029 & 0.0568 & 1281 & 5332 \\
\hline $4 \mathrm{Be}+\mathrm{Fe}_{3} \mathrm{O}_{4}$ & 4.180 & 4482 & 3135 & liquid & l-g & 0.0336 & 0.0188 & 1175 & 4910 \\
\hline $2 \mathrm{Be}+\mathrm{MnO}_{2}$ & 3.882 & 6078 & 2969 & liquid & gas & 0.9527 & 0.5234 & 1586 & 6158 \\
\hline $2 \mathrm{Be}+\mathrm{PbO}_{2}$ & 7.296 & 8622 & 4123 & l-g & gas & 0.4665 & 0.8250 & 875.5 & 6387 \\
\hline $4 \mathrm{Be}+\mathrm{Pb}_{3} \mathrm{O}_{4}$ & 7.610 & 5673 & 3559 & liquid & gas & 0.4157 & 0.8614 & 567.8 & 4322 \\
\hline $2 \mathrm{Be}+\mathrm{SiO}_{2}$ & 2.410 & 2580 & 2482 & solid & liquid & 0.0000 & 0.0000 & 936.0 & 2256 \\
\hline $3 \mathrm{Hf}+2 \mathrm{~B}_{2} \mathrm{O}_{3}$ & 6.125 & 2656 & 2575 & solid & liquid & 0.0000 & 0.0000 & 296.5 & 1816 \\
\hline $3 \mathrm{Hf}+2 \mathrm{Cr}_{2} \mathrm{O}_{3}$ & 7.971 & 2721 & 2572 & solid & liquid & 0.0000 & 0.0000 & 302.3 & 2410 \\
\hline $\mathrm{Hf}+2 \mathrm{CuO}$ & 8.332 & 5974 & 2843 & solid & l-g & 0.3881 & 0.2466 & 567.6 & 4730 \\
\hline $3 \mathrm{Hf}+2 \mathrm{Fe}_{2} \mathrm{O}_{3}$ & 7.955 & 5031 & 2843 & solid & l-g & 0.2117 & 0.1183 & 473.3 & 3765 \\
\hline $2 \mathrm{Hf}+\mathrm{Fe}_{3} \mathrm{O}_{4}$ & 7.760 & 4802 & 2843 & 'solid & $1-g$ & 0.1835 & 0.1025 & 450.4 & 3496 \\
\hline $\mathrm{Hf}+\mathrm{MnO}_{2}$ & 8.054 & 5644 & 3083 & s-1 & gas & 0.3263 & 0.3131 & 534.6 & 4305 \\
\hline $2 \mathrm{Hf}+\mathrm{Pb}_{3} \mathrm{O}_{4}$ & 9.775 & 9382 & 4410 & liquid & gas & 0.2877 & 0.5962 & 345.9 & 3381 \\
\hline $\mathrm{Hf}+\mathrm{SiO}_{2}$ & 6.224 & 2117 & 1828 & solid & liquid & 0.0000 & 0.0000 & 203.3 & 1265 \\
\hline $2 \mathrm{La}+3 \mathrm{AgO}$ & 6.827 & 8177 & 4173 & liquid & gas & 0.4619 & 0.4983 & 646.7 & 4416 \\
\hline $2 \mathrm{La}+3 \mathrm{CuO}$ & 6.263 & 6007 & 2843 & liquid & $1-g$ & 0.3737 & 0.2374 & 606.4 & 3798 \\
\hline $2 \mathrm{La}+\mathrm{Fe}_{2} \mathrm{O}_{3}$ & 5.729 & 4590 & 3135 & liquid & $\mathrm{lg}$ & 0.1234 & 0.0689 & 529.6 & 3034 \\
\hline $2 \mathrm{La}+3 \mathrm{HgO}$ & 8.962 & 7140 & $\geq 4473^{*}$ & $\log$ & gas & $.32-.43$ & $0.65-1$ & 392.0 & 3513 \\
\hline $10 \mathrm{La}+3 \mathrm{I}_{2} \mathrm{O}_{5}$ & 5.501 & 9107 & $\geq 4473^{*}$ & gas & gas & 0.3347 & 1.0000 & 849.2 & 4672 \\
\hline $4 \mathrm{La}+3 \mathrm{MnO}_{2}$ & 5.740 & 5270 & 3120 & liquid & gas & 0.3674 & 0.2019 & 593.4 & 3406 \\
\hline $2 \mathrm{La}+3 \mathrm{PbO}$ & 8.207 & 4598 & 2609 & liquid & gas & 0.3166 & 0.6561 & 287.4 & 2359 \\
\hline $4 \mathrm{La}+3 \mathrm{PbO}_{2}$ & 7.629 & 7065 & $\geq 4473^{*}$ & gas & gas & 0.3927 & 1.0000 & 518.8 & 3958 \\
\hline $8 \mathrm{La}+3 \mathrm{~Pb}_{3} \mathrm{O}_{4}$ & 7.789 & 5628 & 4049 & liquid & gas & 0.2841 & 0.5886 & 378.6 & 2949 \\
\hline $2 \mathrm{La}+3 \mathrm{PdO}$ & 7.769 & 5635 & 3237 & liquid & $\mathrm{lg}$ & 0.2450 & 0.2606 & 536.2 & 4166 \\
\hline $4 \mathrm{La}+3 \mathrm{WO}_{2}$ & 8.366 & 3826 & 3218 & liquid & solid & 0.0000 & 0.0000 & 361.2 & 3022 \\
\hline $2 \mathrm{La}+\mathrm{WO}_{3}$ & 6.572 & 5808 & 4367 & liquid & liquid & 0.0000 & 0.0000 & 445.8 & 2930 \\
\hline $6 \mathrm{Li}+\mathrm{B}_{2} \mathrm{O}_{3}$ & 0.891 & 2254 & 1843 & s-1 & solid & 0.0000 & 0.0000 & 1293 & 1152 \\
\hline $6 \mathrm{Li}+\mathrm{Cr}_{2} \mathrm{O}_{3}$ & 1.807 & 2151 & 1843 & $s-1$ & solid & 0.0000 & 0.0000 & 799.5 & 1445 \\
\hline $2 \mathrm{Li}+\mathrm{CuO}$ & 2.432 & 4152 & 2843 & liquid & l-g & 0.2248 & 0.1428 & 1125 & 2736 \\
\hline $6 \mathrm{Li}+\mathrm{Fe}_{2} \mathrm{O}_{3}$ & 1.863 & 3193 & 2510 & liquid & liquid & 0.0000 & 0.0000 & 1143 & 2130 \\
\hline $8 \mathrm{Li}+\mathrm{Fe}_{3} \mathrm{O}_{4}$ & 0.517 & 3076 & 2412 & liquid & liquid & 0.0000 & 0.0000 & 1053 & 2036 \\
\hline $4 \mathrm{Li}+\mathrm{MnO}_{2}$ & 1.656 & 3336 & 2334 & liquid & l-g & 0.4098 & 0.2251 & 1399 & 2317 \\
\hline $6 \mathrm{Li}+\mathrm{MoO}_{3}$ & 1.688 & 4035 & 2873 & $1-g$ & solid & 0.2155 & 0.0644 & 1342 & 2265 \\
\hline $8 \mathrm{Li}+\mathrm{Pb}_{3} \mathrm{O}_{4}$ & 4.133 & 4186 & 2873 & l-g & liquid. & 0.1655 & 0.0496 & 536.7 & 2218 \\
\hline $4 \mathrm{Li}+\mathrm{SiO}_{2}$ & 1.177 & 1712 & 1687 & solid & s-1 & 0.0000 & 0.0000 & 763.9 & 898.7 \\
\hline $6 \mathrm{Li}+\mathrm{WO}_{3}$ & 2.478 & 3700 & 2873 & $1-g$ & solid & 0.0113 & 0.0034 & 825.4 & 2046 \\
\hline $3 \mathrm{Mg}+\mathrm{B}_{2} \mathrm{O}_{3}$ & 1.785 & 6389 & 3873 & $1-g$ & liquid & 0.4981 & 0.2007 & 2134 & 1195 \\
\hline $3 \mathrm{Mg}+\mathrm{Cr}_{2} \mathrm{O}_{3}$ & 3.164 & 3788 & 2945 & solid & l-g & 0.1023 & 0.0532 & 813.1 & 2573 \\
\hline
\end{tabular}

- More data needed for this calculation. 
Table 1 - Thermite Reactions (cont.)

\begin{tabular}{|c|c|c|c|c|c|c|c|c|c|}
\hline \multicolumn{2}{|c|}{ reactants } & \multicolumn{2}{|c|}{$\begin{array}{c}\text { adiabatic reaction } \\
\text { temperature (K) }\end{array}$} & \multicolumn{2}{|c|}{ state of products } & \multicolumn{2}{|c|}{ gas production } & \multicolumn{2}{|c|}{ heat of reaction } \\
\hline constituents & $\begin{array}{l}\rho_{\mathrm{TMD}}, \\
\mathrm{g} / \mathrm{cm}^{3}\end{array}$ & $\begin{array}{l}\text { w/o phase } \\
\text { changes }\end{array}$ & $\begin{array}{l}\text { w/ phase } \\
\text { changes }\end{array}$ & $\begin{array}{l}\text { state of } \\
\text { oxide }\end{array}$ & $\begin{array}{c}\text { state of } \\
\text { metal }\end{array}$ & $\begin{array}{l}\text { moles gas } \\
\text { per } 100 \mathrm{~g}\end{array}$ & $\begin{array}{l}\text { g of gas } \\
\text { per } g\end{array}$ & $\begin{array}{l}-Q, \\
\text { cal/g }\end{array}$ & $\begin{array}{c}-Q \\
\mathrm{cal} / \mathrm{cm}^{3}\end{array}$ \\
\hline $\mathrm{Mg}+\mathrm{CuO}$ & 3.934 & 6502 & 2843 & solid & $1-g$ & 0.8186 & 0.5201 & 1102 & 44336 \\
\hline $3 \mathrm{Mg}+\mathrm{Fe}_{2} \mathrm{O}_{3}$ & 3.224 & 4703 & 3135 & liquid & $1-g$ & 0.2021 & 0.1129 & 1110 & 3579 \\
\hline $4 \mathrm{Mg}+\mathrm{Fe}_{3} \mathrm{O}_{4}$ & 3.274 & 4446 & 3135 & liquid & $1-g$ & 0.1369 & 0.0764 & 1033 & 3383 \\
\hline $2 \mathrm{Mg}+\mathrm{MnO}_{2}$ & 2.996 & 5209 & 3271 & liquid & gas & 0.7378 & 0.4053 & 1322 & 3961 \\
\hline $4 \mathrm{Mg}+\mathrm{Pb}_{3} \mathrm{O}_{4}$ & 5.965 & 5883 & 3873 & l-g & gas & 0.4216 & 0.8095 & 556.0 & 3316 \\
\hline $2 \mathrm{Mg}+\mathrm{SiO}_{2}$ & 2.148 & 3401 & 2628 & solid & $1-g$ & 0.9200 & $0-.26$ & 789.6 & 1695 \\
\hline $2 \mathrm{Nd}+3 \mathrm{AgO}$ & 7.244 & 7628 & 3602 & liquid & gas & 0.4544 & 0.4902 & 625.9 & 4534 \\
\hline $2 \mathrm{Nd}+3 \mathrm{CuO}$ & 6.719 & 5921 & 2843 & liquid & $1-g$ & 0.3699 & 0.2350 & 603.4 & 4054 \\
\hline $2 \mathrm{Nd}+3 \mathrm{HgO}$ & 9.430 & 7020 & $<5374^{*}$ & gas & gas & 0.4263 & 1.0000 & 392.7 & 3703 \\
\hline $10 \mathrm{Nd}+3 \mathrm{I}_{2} \mathrm{O}_{5}$ & 5.896 & 10067 & $<7580^{*}$ & gas & gas & 0.3273 & 1.0000 & 840.6 & 4956 \\
\hline $4 \mathrm{Nd}+3 \mathrm{MnO}_{2}$ & 6.241 & 5194 & 3287 & liquid & gas & 0.3580 & 0.1967 & 589.9 & 3682 \\
\hline $4 \mathrm{Nd}+3 \mathrm{PbO}_{2}$ & 8.148 & 6938 & $<5284^{*}$ & gas & gas & 0.3862 & 1.0000 & 517.8 & 4219 \\
\hline $8 \mathrm{Nd}+3 \mathrm{~Pb}_{3} \mathrm{O}_{4}$ & 8.218 & 5553 & 3958 & liquid & gas & 0.2803 & 0.5808 & 379.6 & 3120 \\
\hline $2 \mathrm{Nd}+3 \mathrm{PdO}$ & 8.297 & 6197 & 3237 & liquid & $1-g$ & 0.2394 & 0.2547 & 532.7 & 4420 \\
\hline $4 \mathrm{Nd}+3 \mathrm{WO}_{2}$ & 9.016 & 4792 & 3778 & liquid & liquid & 0.0000 & 0.0000 & 362.9 & 3272 \\
\hline $2 \mathrm{Nd}+\mathrm{WO}_{3}$ & 7.074 & 5438 & 4245 & liquid & liquid & 0.0000 & 0.0000 & 446.1 & 3156 \\
\hline $2 \mathrm{Ta}+5 \mathrm{AgO}$ & 9.341 & 6110 & 2436 & liquid & $1-g$ & 0.4229 & 0.4562 & 466.2 & 4355 \\
\hline $2 \mathrm{Ta}+5 \mathrm{CuO}$ & 9.049 & 4044 & 2843 & liquid & $1-g$ & 0.0776 & 0.0493 & 390.3 & 3532 \\
\hline $6 \mathrm{Ta}+5 \mathrm{Fe}_{2} \mathrm{O}_{3}$ & 9.185 & 2383 & 2138 & solid & liquid & 0.0000 & 0.0000 & 235.0 & 2558 \\
\hline $2 \mathrm{Ta}+5 \mathrm{HgO}$ & 12.140 & 5285 & $\leq 4199^{*}$ & liquid & gas & 0.3460 & 0.6942 & 263.3 & 3120 \\
\hline $2 \mathrm{Ta}+\mathrm{I}_{2} \mathrm{O}_{5}$ & 7.615 & 8462 & 7240 & gas & gas & 0.2875 & 1.0000 & 648.6 & 4939 \\
\hline $2 \mathrm{Ta}+5 \mathrm{PbO}$ & 10.640 & 2752 & 2019 & solid & l-g & 0.1475 & 0.3056 & 154.5 & 1644 \\
\hline $4 \mathrm{Ta}+5 \mathrm{PbO}_{2}$ & 11.215 & 4935 & 3472 & liquid & gas & 0.2604 & 0.5397 & 338.6 & 3797 \\
\hline $8 \mathrm{Ta}+5 \mathrm{~Pb}_{3} \mathrm{O}_{4}$ & 10.510 & 3601 & 2019 & solid & $1-g$ & 0.2990 & 0.6196 & 225.0 & 2365 \\
\hline $2 \mathrm{Ta}+5 \mathrm{PdO}$ & 11.472 & 4344 & 3237 & liquid & $1-g$ & 0.0575 & 0.0612 & 360.4 & 4135 \\
\hline $4 \mathrm{Ta}+5 \mathrm{WO}_{2}$ & 13.515 & 2556 & 2196 & liquid & solid & 0.0000 & 0.0000 & 145.1 & 1962 \\
\hline $6 \mathrm{Ta}+5 \mathrm{WO}_{3}$ & 9.876 & 2883 & 2633 & liquid & solid & 0.0000 & 0.0000 & 206.2 & 2036 \\
\hline $3 \mathrm{Th}+2 \mathrm{~B}_{2} \mathrm{O}_{3}$ & 6.688 & 3959 & 3135 & solid & liquid & 0.0000 & 0.0000 & 337.8 & 2259 \\
\hline $3 \mathrm{Th}+2 \mathrm{Cr}_{2} \mathrm{O}_{3}$ & 8.300 & 4051 & 2945 & solid & $\operatorname{l-g}$ & 0.0590 & 0.0307 & 334.5 & 2776 \\
\hline $\mathrm{Th}+2 \mathrm{CuO}$ & 8.582 & 7743 & 2843 & solid & $\mathrm{I}-\mathrm{g}$ & 0.4301 & 0.3421 & 558.7 & 4795 \\
\hline $3 \mathrm{Th}+2 \mathrm{Fe}_{2} \mathrm{O}_{3}$ & 8.280 & 6287 & 3135 & solid & l-g & 0.2619 & 0.1463 & 477.9 & 3957 \\
\hline $2 \mathrm{Th}+\mathrm{Fe}_{3} \mathrm{O}_{4}$ & 8.092 & 5912 & 3135 & solid & l-g & 0.2257 & 0.1261 & 458.5 & 3710 \\
\hline $\mathrm{Th}+\mathrm{MnO}_{2}$ & 8.391 & 7151 & 3910 & liquid & gas & 0.3135 & 0.1722 & 529.2 & 4440 \\
\hline $\mathrm{Th}+\mathrm{PbO}_{2}$ & 10.19 & 10612 & 4673 & l-g & gas & 0.2817 & 0.6231 & 482.8 & 4922 \\
\hline $2 \mathrm{Th}+\mathrm{Pb}_{3} \mathrm{O}_{4}$ & 9.845 & 8532 & 4673 & $1-g$ & gas & 0.2695 & 0.5633 & 360.5 & 3549 \\
\hline $\mathrm{Th}+\mathrm{SiO}_{2}$ & 6.732 & 3813 & 2628 & solid & $1-g$ & $0-.34$ & $0-.10$ & 258.2 & 1738 \\
\hline $3 \mathrm{Ti}+2 \mathrm{~B}_{2} \mathrm{O}_{3}$ & 2.791 & 1498 & 1498 & solid & solid & 0.0000 & 0.0000 & 276.6 & 772.0 \\
\hline $3 \mathrm{Ti}+2 \mathrm{Cr}_{2} \mathrm{O}_{3}$ & 4.959 & 1814 & 1814 & solid. & solid & 0.0000 & 0.0000 & 296.2 & 1469 \\
\hline $\mathrm{Ti}+2 \mathrm{CuO}$ & 5.830 & 5569 & 2843 & liquid & $1-g$ & 0.3242 & 0.2060 & 730.5 & 4259 \\
\hline
\end{tabular}

- More data needed for this calculation. 
Table 1 - Thermite Reactions (cont.)

\begin{tabular}{|c|c|c|c|c|c|c|c|c|c|}
\hline \multicolumn{2}{|c|}{ reactants } & \multicolumn{2}{|c|}{$\begin{array}{c}\text { adiabatic reaction } \\
\text { temperature }(K)\end{array}$} & \multicolumn{2}{|c|}{ state of products } & \multicolumn{2}{|c|}{ gas production } & \multicolumn{2}{|c|}{ heat of reaction } \\
\hline constituents & $\begin{array}{l}\rho_{\mathrm{TMD}}, \\
\mathrm{g} / \mathrm{cm}^{3}\end{array}$ & $\begin{array}{l}\text { w/o phase } \\
\text { changes }\end{array}$ & $\begin{array}{l}\text { w/ phase } \\
\text { changes }\end{array}$ & $\begin{array}{l}\text { state of } \\
\text { oxide }\end{array}$ & $\begin{array}{l}\text { state of } \\
\text { metal }\end{array}$ & $\begin{array}{l}\text { moles gas } \\
\text { per } 100 \mathrm{~g}\end{array}$ & $\begin{array}{l}\mathrm{g} \text { of gas } \\
\text { per } \mathrm{g}\end{array}$ & $\begin{array}{l}-Q, \\
\mathrm{cal} / \mathrm{g}\end{array}$ & $\begin{array}{c}-Q, \\
\mathrm{cal} / \mathrm{cm}^{3}\end{array}$ \\
\hline $3 \mathrm{Ti}+2 \mathrm{Fe}_{2} \mathrm{O}_{3}$ & 5.010 & 3358 & 2614 & liquid & liquid & 0.0000 & 0.0000 & 612.0 & 3066 \\
\hline $\mathrm{Ti}+\mathrm{Fe}_{3} \mathrm{O}_{4}$ & 4.974 & 3113 & 2334 & liquid & liquid & 0.0000 & 0.0000 & 563.0 & 2800 \\
\hline $\mathrm{Ti}+\mathrm{MnO}_{2}$ & 4.826 & 3993 & 2334 & liquid & l-g & 0.3783 & 0.2078 & 752.7 & 3633 \\
\hline $2 \mathrm{Ti}+\mathrm{Pb}_{3} \mathrm{O}_{4}$ & 8.087 & 5508 & 2498 & liquid & gas & 0.3839 & 0.7955 & 358.1 & 2896 \\
\hline $\mathrm{Ti}+\mathrm{SiO}_{2}$ & 3.241 & 715 & 715 & solid & solid & 0.0000 & 0.0000 & 75.0 & 243.1 \\
\hline $2 \mathrm{Y}+3 \mathrm{CuO}$ & 5.404 & 7668 & 3124 & liquid & $\mathrm{l}-\mathrm{g}$ & 0.7204 & 0.4577 & 926.7 & 5008 \\
\hline $8 \mathrm{Y}+3 \mathrm{Fe}_{3} \mathrm{O}_{4}$ & 4.803 & 5791 & 3135 & liqquid & $1-g$ & 0.3812 & 0.2129 & 856.3 & 4113 \\
\hline $10 \mathrm{Y}+3 \mathrm{I}_{2} \mathrm{O}_{5}$ & 4.638 & 12416 & $>4573^{*}$ & gas & gas & 0.4231 & 1.0000 & 1144 & 5308 \\
\hline $4 \mathrm{Y}+3 \mathrm{MnO}_{2}$ & 4.690 & 7405 & $<5731^{*}$ & gas & gas & 0.8110 & 1.0000 & 1022 & 4792 \\
\hline $2 \mathrm{Y}+\mathrm{MoO}_{3}$ & 4.567 & 8778 & $\geq 4573^{*}$ & gas & liquid & 0.6215 & 1.0000 & 1005 & 4589 \\
\hline $2 \mathrm{Y}+\mathrm{Ni}_{2} \mathrm{O}_{3}$ & 4.636 & 7614 & 3955 & liquid & gas & 0.5827 & 0.3420 & 1120 & 5194 \\
\hline $4 \mathrm{Y}+3 \mathrm{PbO}_{2}$ & 6.875 & 9166 & $\geq 4573^{*}$ & gas & gas & 0.4659 & 1.0000 & 751.0 & 5163 \\
\hline $2 Y+3 P d O$ & 7.020 & 8097 & 3237 & liquid & l-g & 0.4183 & 0.4451 & 768.1 & 5371 \\
\hline $4 \mathrm{Y}+3 \mathrm{SnO}_{2}$ & 5.604 & 7022 & 4573 & $\log$ & gas & $.37-.62$ & $0.44-1$ & 726.1 & 4068 \\
\hline $10 \mathrm{Y}+3 \mathrm{Ta}_{2} \mathrm{O}_{5}$ & 6.316 & 5564 & $\geq 4573^{*}$ & l-g & liquid & $0-0.23$ & $0-0.51$ & 469.7 & 2966 \\
\hline $10 \mathrm{Y}+3 \mathrm{~V}_{2} \mathrm{O}_{5}$ & 3.970 & 7243 & $\geq 3653^{*}$ & l-g & gas & 0.2130 & 0.4181 & 972.5 & 3861 \\
\hline $2 \mathrm{Y}+\mathrm{WO}_{3}$ & 5.677 & 8296 & $\geq 4573 *$ & gas & liquid & 0.2441 & 0.5512 & 732.2 & 4157 \\
\hline $3 \mathrm{Zr}+2 \mathrm{~B}_{2} \mathrm{O}_{3}$ & 3.782 & 2730 & 2573 & solid & s-1 & 0.2930 & 0.0317 & 437.4 & 1654 \\
\hline $3 \mathrm{Zr}+2 \mathrm{Cr}_{2} \mathrm{O}_{3}$ & 5.713 & 2915 & 2650 & solid & liquid & 0.0000 & 0.0000 & 423.0 & 2417 \\
\hline $\mathrm{Zr}+2 \mathrm{CuO}$ & 6.400 & 6103 & 2843 & solid & 1-g & 0.5553 & 0.3529 & 752.9 & 4818 \\
\hline $3 \mathrm{Zr}+2 \mathrm{Fe}_{2} \mathrm{O}_{3}$ & 5.744 & 4626 & 3135 & liquid & l-g & 0.0820 & 0.0458 & 666.2 & 3827 \\
\hline $2 \mathrm{Zr}+\mathrm{Fe}_{3} \mathrm{O}_{4}$ & 5.668 & 4103 & 3135 & liquid & $\mathrm{l-g}$ & 0.0277 & 0.0155 & 625.1 & 3543 \\
\hline $\mathrm{Zr}+\mathrm{MnO}_{2}$ & 5.647 & 5385 & 2983 & $\mathrm{~s}-1$ & gas & 0.5613 & 0.3084 & 778.7 & 4398 \\
\hline $2 \mathrm{Zr}+\mathrm{Pb}_{3} \mathrm{O}_{4}$ & 8.359 & 6595 & 3300 & l-g & gas & 0.3683 & 0.7440 & 408.1 & 3412 \\
\hline $\mathrm{Zr}+\mathrm{SiO}_{2}$ & 4.098 & 2233 & 1687 & solid & $\mathrm{s}-1$ & 0.0000 & 0.0000 & 299.7 & 1228 \\
\hline
\end{tabular}

- More data needed for this calculation. 
Table 2 - Intermetallic Reactions

\begin{tabular}{|c|c|c|c|c|c|c|c|c|}
\hline \multicolumn{2}{|c|}{ reactants } & \multicolumn{2}{|c|}{$\begin{array}{c}\text { adiabatic reaction } \\
\text { temperature }(K)\end{array}$} & \multirow{2}{*}{$\begin{array}{c}\text { state of } \\
\text { intermetallic } \\
\text { product }\end{array}$} & \multicolumn{2}{|c|}{ gas production } & \multicolumn{2}{|c|}{ heat of reaction } \\
\hline constituents & $\begin{array}{l}\rho_{\mathrm{TMD}} \\
\mathrm{g} / \mathrm{cm}^{3}\end{array}$ & $\begin{array}{l}\text { w/o phase } \\
\text { changes }\end{array}$ & $\begin{array}{l}\text { w/ phase } \\
\text { changes }\end{array}$ & & $\begin{array}{l}\text { moles gas } \\
\text { per } 100 \mathrm{~g}\end{array}$ & $\begin{array}{l}\text { g gas } \\
\text { per g }\end{array}$ & $\begin{array}{c}-\mathrm{Q}, \\
\mathrm{cal} / \mathrm{g}\end{array}$ & $\begin{array}{c}-Q \\
\mathrm{cal} / \mathrm{cm}^{3}\end{array}$ \\
\hline $\mathrm{Al}+2 \mathrm{~B}$ & 2.607 & 2251 & $\geq 1253^{*}$ & $1-g$ & $0-2.1$ & $0-1$ & 742 & 1940 \\
\hline $4 \mathrm{Al}+3 \mathrm{C}$ & 2.574 & 1673 & 1673 & solid & 0.0 & 0.0 & 371 & 965 \\
\hline $2 \mathrm{Al}+\mathrm{Ca}$ & 2.051 & 2836 & 1738 & liquid & 0.0 & 0.0 & 558 & 1140 \\
\hline $4 \mathrm{Al}+\mathrm{Ca}$ & 2.248 & 1880 & $\geq 973^{*}$ & $\mathrm{~s}-1$ & 0.0 & 0.0 & 348 & 782 \\
\hline $4 \mathrm{Al}+\mathrm{Ce}$ & 4.095 & 1173 & 1173 & solid & 0.0 & 0.0 & 126 & 458 \\
\hline $\mathrm{Al}+\mathrm{Co}$ & 5.171 & 2195 & $\geq 1913^{*}$ & $\mathrm{~s}-1$ & 0.0 & 0.0 & 307 & 1590 \\
\hline $4 \mathrm{Al}+\mathrm{Co}$ & 3.581 & $*$ & $*$ & $*$ & $*$ & $*$ & 231 & 637 \\
\hline $5 \mathrm{Al}+2 \mathrm{Co}$ & 3.999 & 1755 & $\geq 1453^{*}$ & $s-1$ & 0.0 & 0.0 & 277 & 1110 \\
\hline $3 \mathrm{Al}+\mathrm{Cr}$ & 3.568 & 793 & 793 & solid & 0.0 & 0.0 & 120 & 430 \\
\hline $\mathrm{Al}+\mathrm{Cu}$ & 5.294 & 935 & 935 & solid & 0.0 & 0.0 & 108 & 573 \\
\hline $\mathrm{Al}+\mathrm{Fe}$ & 4.844 & 1423 & 1423 & solid & 0.0 & 0.0 & 211 & 1020 \\
\hline $3 \mathrm{Al}+\mathrm{Fe}$ & 3.688 & 1407 & 1407 & solid & 0.0 & 0.0 & 278 & 1020 \\
\hline $4 \mathrm{Al}+\mathrm{La}$ & 3.946 & 1495 & $*$ & $s-1$ & 0.0 & 0.0 & 166 & 780 \\
\hline $\mathrm{Al}+\mathrm{Li}$ & 1.476 & 1160 & $\geq 973^{*}$ & $\mathrm{~s}-\mathrm{I}$ & 0.0 & 0.0 & 345 & 509 \\
\hline $\mathrm{Al}+\mathrm{Mn}$ & 4.676 & 803 & 803 & solid & 0.0 & 0.0 & 124 & 586 \\
\hline $\mathrm{Al}+\mathrm{Ni}$ & 5.165 & 2362 & $\geq 1911^{*}$ & $s-1$ & 0.0 & 0.0 & 330 & 1710 \\
\hline $\mathrm{Al}+3 \mathrm{Ni}$ & 6.820 & 1524 & 1524 & solid & 0.0 & 0.0 & 180 & 1230 \\
\hline $\mathrm{Al}+\mathrm{Pd}$ & 7.072 & 2725 & 2653 & liquid & 0.0 & 0.0 & 327 & 2890 \\
\hline $4 \mathrm{Al}+\mathrm{Pr}$ & 4.094 & 1703 & $*$ & s-l & 0.0 & 0.0 & 216 & 800 \\
\hline $\mathrm{Al}+\mathrm{Pt}$ & 11.63 & 3379 & 3073 & liquid & 0.0 & 0.0 & 216 & 2510 \\
\hline $4 \mathrm{Al}+\mathrm{Pu}$ & 6.708 & 1403 & 1403 & solid & 0.0 & 0.0 & 123 & $: \quad 820$ \\
\hline $2 \mathrm{Al}+3 \mathrm{~S}$ & 2.102 & $*$ & $*$ & $*$ & 0.0 & 0.0 & 800 & 1680 \\
\hline $\mathrm{Al}+\mathrm{Ta}$ & 9.952 & 1011 & 1011 & solid & 0.0 & 0.0 & 56.7 & 564 \\
\hline $3 \mathrm{Al}+\mathrm{Ta}$ & 6.407 & 665 & 665 & solid & 0.0 & 0.0 & 35.9 & 230 \\
\hline $\mathrm{Al}+\mathrm{Ti}$ & 3.628 & 1597 & 1597 & solid & 0.0 & 0.0 & 240 & 872 \\
\hline $\mathrm{Al}+3 \mathrm{Ti}$ & 4.071 & $*$ & $*$ & $*$ & $*$ & $*$ & 138 & 560 \\
\hline $2 \mathrm{Al}+\mathrm{Ti}$ & 3.326 & 1643 & $*$ & $1-s$ & $*$ & $*$ & 314 & 1100 \\
\hline $3 \mathrm{Al}+\mathrm{Ti}$ & 3.172 & 1591 & 1591 & solid & 0.0 & 0.0 & 272 & 862 \\
\hline $3 \mathrm{Al}+2 \mathrm{Ti}$ & 3.448 & $*$ & $*$ & $*$ & $*$ & $*$ & 158 & 544 \\
\hline $4 \mathrm{Al}+\mathrm{U}$ & 6.582 & 1205 & $*$ & liquid & 0.0 & 0.0 & 89.8 & 591 \\
\hline $3 \mathrm{Al}+\mathrm{V}$ & 3.412 & 1023 & 1023 & solid & 0.0 & 0.0 & 198 & 792 \\
\hline $2 \mathrm{Al}+\mathrm{Zr}$ & 4.240 & 1923 & $*$ & l-s & $*$ & $*$ & 267 & 1130 \\
\hline $4 B+C$ & 2.444 & 1202 & 1202 & solid & 0.0 & 0.0 & 308 & 751 \\
\hline $6 \mathrm{~B}+\mathrm{Ce}$ & 4.374 & 2388 & 2388 & solid & 0.0 & 0.0 & 395 & 1730 \\
\hline $2 \mathrm{~B}+\mathrm{Cr}$ & 4.622 & 1571 & 1571 & solid & 0.0 & 0.0 & 306 & 1410 \\
\hline $2 \mathrm{~B}+\mathrm{Hf}$ & 8.232 & 3945 & 3653 & liquid & 0.0 & 0.0 & 401 & 3300 \\
\hline $6 \mathrm{~B}+\mathrm{La}$ & 4.198 & 2503 & $*$ & l-s & 0.0 & 0.0 & 560 & 2350 \\
\hline $2 \mathrm{~B}+\mathrm{Mg}$ & 2.031 & 1706 & 1706 & $\operatorname{dec}$ & 0.0 & 0.0 & 479 & 972 \\
\hline $6 \mathrm{~B}+\mathrm{Mg}$ & 2.234 & 918 & 918 & solid & 0.0 & 0.0 & 251 & 600 \\
\hline
\end{tabular}

- More data needed for this calculation. 
Table 2 - Intermetallic Reactions (cont.)

\begin{tabular}{|c|c|c|c|c|c|c|c|c|}
\hline \multicolumn{2}{|c|}{ reactants } & \multicolumn{2}{|c|}{$\begin{array}{l}\text { adiabatic reaction } \\
\text { temperature (K) }\end{array}$} & \multirow{2}{*}{$\begin{array}{c}\text { state of } \\
\text { intermetallic } \\
\text { product }\end{array}$} & \multicolumn{2}{|c|}{ gas production } & \multicolumn{2}{|c|}{ heat of reaction } \\
\hline constituents & $\begin{array}{l}\rho_{\mathrm{TMM}} \\
\mathrm{g} / \mathrm{cm}^{3}\end{array}$ & $\begin{array}{l}\text { w/o phase } \\
\text { changes }\end{array}$ & $\begin{array}{l}\text { w/ phase } \\
\text { changes }\end{array}$ & & $\begin{array}{l}\text { moles gas } \\
\text { per } 100 \mathrm{~g}\end{array}$ & $\begin{array}{l}\text { g gas } \\
\text { per } g\end{array}$ & $\begin{array}{l}-Q \\
\mathrm{cal} / \mathrm{g}\end{array}$ & $\begin{array}{c}-Q \\
\mathrm{cal} / \mathrm{cm}^{3}\end{array}$ \\
\hline $2 \mathrm{~B}+\mathrm{Mn}$ & 4.732 & 1386 & 1386 & solid & 0.0 & 0.0 & 294 & 1390 \\
\hline $2 \mathrm{~B}+\mathrm{Mo}$ & 6.091 & 1533 & 1533 & solid & 0.0 & 0.0 & 196 & 1280 \\
\hline $2 \mathrm{~B}+\mathrm{Nb}$ & 5.875 & 2793 & 2793 & solid & 0.0 & 0.0 & 524 & 3080 \\
\hline $6 \mathrm{~B}+\mathrm{Sm}$ & 4.684 & 1233 & 1233 & solid & 0.0 & 0.0 & 232 & 1050 \\
\hline $6 \mathrm{~B}+\mathrm{Si}$ & 2.497 & 503 & 503 & solid & 0.0 & 0.0 & 76.4 & 177 \\
\hline $2 \mathrm{~B}+\mathrm{Ta}$ & 10.36 & 2766 & 2766 & solid & 0.0 & 0.0 & 247 & 2560 \\
\hline $4 B+T h$ & 7.240 & 1823 & 1823 & solid & 0.0 & 0.0 & 189 & 1360 \\
\hline$B+T i$ & 3.922 & 3559 & $\geq 2453^{*}$ & 1 or $g$ & $0-1.7$ & $0-1$ & 652 & 2560 \\
\hline $2 \mathrm{~B}+\mathrm{Ti}$ & 3.603 & 3710 & 3498 & liquid & 0.0 & 0.0 & 1320 & 5170 \\
\hline $2 B+U$ & 12.220 & 1335 & 1335 & solid & 0.0 & 0.0 & 149 & 1820 \\
\hline $4 \mathrm{~B}+U$ & 9.407 & 2124 & 2124 & solid & 0.0 & 0.0 & 209 & 1960 \\
\hline$B+V$ & 4.749 & 2574 & 2574 & solid & 0.0 & 0.0 & 536 & 2540 \\
\hline $2 B+V$ & 4.187 & 2960 & 2960 & $\mathrm{~s}-1$ & 0.0 & 0.0 & 671 & 2810 \\
\hline $5 B+2 W$ & 10.37 & 1233 & 1233 & solid & 0.0 & 0.0 & 83 & 1350 \\
\hline $6 B+Y$ & 3.354 & 973 & 973 & solid & 0.0 & 0.0 & 156 & 503 \\
\hline $2 \mathrm{~B}+\mathrm{Zr}$ & 4.926 & 3783 & 3673 & liquid & 0.0 & 0.0 & 683 & 3360 \\
\hline $3 \mathrm{Ba}+2 \mathrm{Bi}$ & 5.185 & 1673 & $*$ & liquid & 0.0 & 0.0 & 87.3 & 169 \\
\hline $\mathrm{Ba}+2 \mathrm{C}$ & 3.236 & 1466 & 1466 & solid & 0.0 & 0.0 & 111 & 359 \\
\hline $2 \mathrm{Ba}+\mathrm{Pb}$ & 4.025 & 1913 & $\geq 1201^{*}$ & $1-g$ & $0-.21$ & $0-1$ & 143 & 576 \\
\hline $3 \mathrm{Ba}+2 \mathrm{Sb}$ & 4.252 & 1833 & * & liquid & $*$ & $*$ & 133 & 563 \\
\hline $2 \mathrm{Ba}+\mathrm{Sn}$ & 4.153 & 2398 & * & l-g & $0-.25$ & $0-1$ & 240 & 1000 \\
\hline $\mathrm{Be}+2 \mathrm{C}$ & 2.131 & 3043 & $*$ & l-g & $0-.33$ & $0-1$ & 1750 & 3720 \\
\hline $2 \mathrm{Be}+\mathrm{C}$ & 1.995 & 1932 & 1932 & solid & 0.0 & 0.0 & 931 & 1860 \\
\hline $5 \mathrm{Be}+\mathrm{Nb}$ & 3.920 & 1663 & 1663 & solid & 0.0 & 0.0 & 336 & 1300 \\
\hline $13 \mathrm{Be}+\mathrm{Pu}$ & 4.756 & 723 & 723 & solid & 0.0 & 0.0 & 100 & 476 \\
\hline $13 \mathrm{Be}+\mathrm{U}$ & 4.679 & 773 & 773 & solid & 0.0 & 0.0 & 110 & 513 \\
\hline $\mathrm{Bi}+\mathrm{K}$ & 3.723 & 1253 & $*$ & liquid & 0.0 & 0.0 & 55 & 204 \\
\hline $\mathrm{Bi}+3 \mathrm{~K}$ & 2.071 & 1791 & $*$ & liquid? & $*$ & $*$ & 127 & 263 \\
\hline $\mathrm{Bi}+\mathrm{Li}$ & 6.319 & 1273 & $\geq 688^{*}$ & liquid & 0.0 & 0.0 & 85.5 & 535 \\
\hline $2 \mathrm{C}+\mathrm{Ca}$ & 1.757 & 1113 & 1113 & solid & 0.0 & 0.0 & 223 & 392 \\
\hline $2 \mathrm{C}+\mathrm{Ce}$ & 5.201 & 1779 & 1779 & solid & 0.0 & 0.0 & 170 & 884 \\
\hline $3 \mathrm{C}+7 \mathrm{Cr}$ & 5.978 & 1175 & 1175 & solid & 0.0 & 0.0 & 136 & 813 \\
\hline $\mathrm{C}+\mathrm{Hf}$ & 9.084 & 4441 & $\geq 4223 *$ & $s-1$ & 0.0 & 0.0 & 315 & 2860 \\
\hline $2 \mathrm{C}+\mathrm{La}$ & 4.905 & 1973 & 1973 & solid & 0.0 & 0.0 & 178 & 870 \\
\hline $3 \mathrm{C}+7 \mathrm{Mn}$ & 6.130 & 742 & 742 & solid & 0.0 & 0.0 & 62.1 & 380 \\
\hline $\mathrm{C}+2 \mathrm{Mo}$ & 7.662 & 1077 & 1077 & solid & 0.0 & 0.0 & 62.3 & 477 \\
\hline $2 \mathrm{C}+2 \mathrm{Na}$ & 1.206 & 733 & 733 & solid & 0.0 & 0.0 & 137 & 165 \\
\hline $0.98 \mathrm{C}+\mathrm{Nb}$ & 6.522 & 3182 & 3182 & solid & 0.0 & 0.0 & 317 & 2070 \\
\hline $\mathrm{C}+\mathrm{Nb}$ & 9.678 & 3003 & 3003 & solid & 0.0 & 0.0 & 321 & 2060 \\
\hline $\mathrm{C}+2 \mathrm{Nb}$ & 7.328 & 2634 & 2634 & solid & 0.0 & 0.0 & 230 & 1680 \\
\hline
\end{tabular}

More data needed for this calculation. 
Table 2 - Intermetallic Reactions (cont.)

\begin{tabular}{|c|c|c|c|c|c|c|c|c|}
\hline \multicolumn{2}{|c|}{ reactants } & \multicolumn{2}{|c|}{$\begin{array}{l}\text { adiabatic reaction } \\
\text { temperature }(K)\end{array}$} & \multirow{2}{*}{$\begin{array}{c}\text { state of } \\
\text { intermetallic } \\
\text { product }\end{array}$} & \multicolumn{2}{|c|}{ gas production } & \multicolumn{2}{|c|}{ heat of reaction } \\
\hline constituents & $\begin{array}{l}\rho_{\mathrm{TMD}}, \\
\mathrm{g} / \mathrm{cm}^{3} \\
\end{array}$ & $\begin{array}{l}\text { w/o phase } \\
\text { changes }\end{array}$ & $\begin{array}{l}\text { w/ phase } \\
\text { changes }\end{array}$ & & $\begin{array}{l}\text { moles gas } \\
\text { per } 100 \mathrm{~g}\end{array}$ & $\begin{array}{l}\text { g gas } \\
\text { per g }\end{array}$ & $\begin{array}{l}-Q, \\
\mathrm{cal} / \mathrm{g}\end{array}$ & $\begin{array}{c}-Q, \\
\mathrm{cal} / \mathrm{cm}^{3}\end{array}$ \\
\hline $\mathrm{C}+\mathrm{Si}$ & 2.416 & 1914 & 1914 & solid & 0.0 & 0.0 & 436 & 1050 \\
\hline $2 \mathrm{C}+\mathrm{Sr}$ & 2.518 & 1242 & 1242 & solid & 0.0 & 0.0 & 160 & 404 \\
\hline $\mathrm{C}+\mathrm{Ta}$ & 11.90 & 2678 & 2678 & solid & 0.0 & 0.0 & 179 & 2120 \\
\hline $1.94 \mathrm{C}+\mathrm{Th}$ & 8.230 & 2211 & 2211 & solid & 0.0 & 0.0 & 138 & 1140 \\
\hline $2 \mathrm{C}+\mathrm{Th}$ & 8.169 & 3073 & 3073 & solid & 0.0 & 0.0 & 179 & 1280 \\
\hline $\mathrm{C}+\mathrm{Ti}$ & 3.754 & 3644 & 3523 & liquid & 0.0 & 0.0 & 736 & 2760 \\
\hline$C+U$ & 13.96 & 1871 & 1871 & solid & 0.0 & 0.0 & 93.6 & 1310 \\
\hline $2 \mathrm{C}+\mathrm{U}$ & 11.29 & 1573 & 1573 & solid & 0.0 & 0.0 & 95.5 & 1080 \\
\hline$C+V$ & 4.499 & 2121 & 2121 & solid & 0.0 & 0.0 & 383 & 1720 \\
\hline$C+W$ & 13.20 & 1259 & 1259 & solid & 0.0 & 0.0 & 49.0 & 647 \\
\hline $\mathrm{C}+\mathrm{Zr}$ & 5.276 & 3800 & 3800 & solid & 0.0 & 0.0 & 455 & 2400 \\
\hline $\mathrm{Ca}+2 \mathrm{Mg}$ & 1.649 & 801 & 801 & solid & 0.0 & 0.0 & 108 & 178 \\
\hline $2 \mathrm{Ca}+\mathrm{Pb}$ & 4.105 & 1713 & $\geq 1476^{*}$ & liquid & 0.0 & 0.0 & 172 & 705 \\
\hline $\mathrm{Ca}+\mathrm{Si}$ & 2.490 & 2504 & 2504 & liquid & 0.0 & 0.0 & 529 & 1320 \\
\hline $\mathrm{Ca}+\mathrm{Sn}$ & 3.772 & 3622 & $\geq 1273^{*}$ & 1 or $g$ & $0-1.5$ & $0-1$ & 239 & 903 \\
\hline $2 \mathrm{Ca}+\mathrm{Sn}$ & 2.927 & 2994 & $\geq 1408^{*}$ & lorg & $0-.50$ & $0-1$ & 377 & 1100 \\
\hline $11 \mathrm{Cd}+\mathrm{Pu}$ & 1.056 & 843 & 843 & solid & 0.0 & 0.0 & 31 & 293 \\
\hline $\mathrm{Ce}+\mathrm{Mg}$ & 4.713 & 1552 & $*$ & $s-1$ & 0.0 & 0.0 & 111 & 523 \\
\hline $2 \mathrm{Ce}+\mathrm{Pb}$ & 8.194 & 1653 & $\geq 1653^{*}$ & s-1 & 0.0 & 0.0 & 81.5 & 665 \\
\hline $\mathrm{Ce}+2 \mathrm{Si}$ & 4.547 & 2083 & $*$ & liquid & 0.0 & 0.0 & 255 & 1100 \\
\hline $\mathrm{Ce}+\mathrm{Zn}$ & 6.904 & 1413 & $>1098^{*}$ & liquid & 0.0 & 0.0 & 78 & 535 \\
\hline $\mathrm{Co}+\mathrm{Si}$ & 4.862 & 1733 & $\geq 1733^{*}$ & s-1 & 0.0 & 0.0 & 299 & 1450 \\
\hline $\mathrm{Cr}+\mathrm{Si}$ & 4.316 & 1231 & 1231 & solid & 0.0 & 0.0 & 159 & 684 \\
\hline $\mathrm{Cr}+2 \mathrm{Si}$ & 3.625 & 1530 & 1530 & solid & 0.0 & 0.0 & 222 & 804 \\
\hline $3 \mathrm{Cr}+\mathrm{Si}$ & 5.558 & 1493 & 1493 & solid & 0.0 & 0.0 & 179 & 996 \\
\hline $5 \mathrm{Cr}+3 \mathrm{Si}$ & 4.900 & 1671 & 1671 & solid & 0.0 & 0.0 & 226 & 1110 \\
\hline $\mathrm{Cu}+2 \mathrm{Mg}$ & 3.199 & 665 & 665 & solid & 0.0 & 0.0 & 61.0 & 195 \\
\hline $2 \mathrm{Cu}+\mathrm{Mg}$ & 5.368 & 721 & 721 & solid & 0.0 & 0.0 & 52.9 & 284 \\
\hline $\mathrm{Cu}+\mathrm{Pd}$ & 10.64 & 873 & 873 & solid & 0.0 & 0.0 & 44.5 & 472 \\
\hline $\mathrm{Fe}+\mathrm{Si}$ & 4.564 & 1659 & 1659 & solid & 0.0 & 0.0 & 225 & 1020 \\
\hline $\mathrm{Ge}+2 \mathrm{Mg}$ & 4.123 & 1678 & 1391 & $s-1$ & 0.0 & 0.0 & 207 & 853 \\
\hline $2 \mathrm{Ge}+\mathrm{Nb}$ & 6.269 & 1443 & 1443 & solid & 0.0 & 0.0 & 84.4 & 524 \\
\hline $\mathrm{Li}+\mathrm{Pb}$ & 6.848 & 1258 & $\geq 755^{*}$ & liquid & 0.0 & 0.0 & 67.2 & 460 \\
\hline $\mathrm{Li}+\mathrm{Sb}$ & 4.123 & 1333 & $*$ & liquid & 0.0 & 0.0 & 170 & 700 \\
\hline $\mathrm{Li}+\mathrm{Sn}$ & 4.298 & 1516 & * & s-1 & 0.0 & 0.0 & 134 & 576 \\
\hline $\mathrm{Mg}+\mathrm{S}$ & 2.037 & 7039 & $*$ & $*$ & $*$ & $*$ & 1500 & 3060 \\
\hline $3 \mathrm{Mg}+2 \mathrm{Sb}$ & 4.039 & 1433 & 1433 & solid & 0.0 & 0.0 & 121 & 484 \\
\hline $\mathrm{Mg}+\mathrm{Se}$ & 3.398 & 4817 & $*$ & $*$ & $*$ & $*$ & 678 & 2300 \\
\hline $2 \mathrm{Mg}+\mathrm{Si}$ & 1.956 & 1286 & 1286 & solid & 0.0 & 0.0 & 247 & 483 \\
\hline $2 \mathrm{Mg}+\mathrm{Sn}$ & 3.787 & 1163 & 1163 & solid & 0.0 & 0.0 & 113 & 450 \\
\hline
\end{tabular}

- More data needed for this calculation. 
Table 2 - Intermetallic Reactions (cont.)

\begin{tabular}{|c|c|c|c|c|c|c|c|c|}
\hline \multicolumn{2}{|c|}{ reactants } & \multicolumn{2}{|c|}{$\begin{array}{c}\text { adiabatic reaction } \\
\text { temperature }(K)\end{array}$} & \multirow{2}{*}{$\begin{array}{c}\text { state of } \\
\text { intermetallic } \\
\text { product }\end{array}$} & \multicolumn{2}{|c|}{ gas production } & \multicolumn{2}{|c|}{ heat of reaction } \\
\hline constituents & $\begin{array}{l}\rho_{\mathrm{TMD}} \\
\mathrm{g} / \mathrm{cm}^{3}\end{array}$ & $\begin{array}{l}\text { w/o phase } \\
\text { changes }\end{array}$ & $\begin{array}{l}\text { w/ phase } \\
\text { changes }\end{array}$ & & $\begin{array}{l}\text { moles gas } \\
\text { per } 100 \mathrm{~g}\end{array}$ & $\begin{array}{l}\text { g gas } \\
\text { per g }\end{array}$ & $\begin{array}{l}-Q \\
\mathrm{cal} / \mathrm{g}\end{array}$ & $\begin{array}{c}-Q, \\
\mathrm{cal} / \mathrm{cm}^{3}\end{array}$ \\
\hline $\mathrm{Mg}+\mathrm{Te}$ & 4.311 & 4676 & $*$ & $*$ & $*$ & $*$ & 329 & 1420 \\
\hline $2 \mathrm{Mg}+\mathrm{Th}$ & 5.767 & 1067 & 1067 & solid & 0.0 & 0.0 & 54 & 311 \\
\hline $\mathrm{Mg}+\mathrm{U}$ & 9.874 & 2213 & $*$ & liquid & 0.0 & 0.0 & 157 & 1570 \\
\hline $\mathrm{Mg}+\mathrm{Y}$ & 3.343 & 1943 & $*$ & liquid & 0.0 & 0.0 & 274 & 912 \\
\hline $\mathrm{Mn}+\mathrm{S}$ & 2.370 & 1394 & 1394 & solid & 0.0 & 0.0 & 164 & 390 \\
\hline $\mathrm{Mn}+\mathrm{Si}$ & 4.415 & 1615 & $\geq 1543^{*}$ & $\mathrm{~s}-1$ & 0.0 & 0.0 & 224 & 989 \\
\hline $\mathrm{Mn}+1.7 \mathrm{Si}$ & 3.846 & 1433 & $*$ & $\mathrm{~s}-1$ & 0.0 & 0.0 & 226 & 847 \\
\hline $\mathrm{Mo}+2 \mathrm{Si}$ & 4.581 & 1854 & 1854 & solid & 0.0 & 0.0 & 187 & 855 \\
\hline $\mathrm{Mo}+7 \mathrm{Si}$ & 3.265 & 1823 & 1823 & solid & 0.0 & 0.0 & 206 & 940 \\
\hline $3 \mathrm{Mo}+\mathrm{Si}$ & 7.308 & 1278 & 1278 & solid & 0.0 & 0.0 & 76.9 & 562 \\
\hline $5 \mathrm{Mo}+3 \mathrm{Si}$ & 6.476 & 788 & 788 & solid & 0.0 & 0.0 & 43.1 & 279 \\
\hline $\mathrm{Na}+\mathrm{Sb}$ & 3.453 & 1090 & $*$ & liquid & 0.0 & 0.0 & 110 & 380 \\
\hline $\mathrm{Na}+\mathrm{Sn}$ & 3.548 & 1073 & 1073 & solid & 0.0 & 0.0 & 71.4 & 254 \\
\hline $\mathrm{Nb}+\mathrm{Ni}$ & 8.695 & 1083 & 1083 & solid & 0.0 & 0.0 & 71 & 610 \\
\hline $\mathrm{Nb}+2 \mathrm{Si}$ & 4.463 & 1897 & 1897 & solid & 0.0 & 0.0 & 201 & 898 \\
\hline $5 \mathrm{Nb}+3 \mathrm{Si}$ & 6.233 & 2518 & 2518 & solid & 0.0 & 0.0 & 222 & 1390 \\
\hline $\mathrm{Ni}+\mathrm{Si}$ & 4.855 & 1838 & $>1265^{*}$ & $\mathrm{~s}-1$ & 0.0 & 0.0 & 235 & 1140 \\
\hline $\mathrm{Pd}+\mathrm{Sn}$ & 8.966 & 1599 & $*$ & $s-1$ & 0.0 & 0.0 & 112 & 1000 \\
\hline $\mathrm{Pu}+12 \mathrm{Zn}$ & 8.409 & 973 & 973 & solid & 0.0 & 0.0 & 71.3 & 600 \\
\hline $\mathrm{S}+\mathrm{Zn}$ & 2.419 & 4144 & $*$ & 1-g* & 0.0 & 0.0 & 500 & 1210 \\
\hline $\mathrm{Si}+2 \mathrm{Ta}$ & 11.788 & 1836 & 1836 & solid & 0.0 & 0.0 & 76.9 & 907 \\
\hline $2 \mathrm{Si}+\mathrm{Ta}$ & 7.086 & 1781 & 1781 & solid & 0.0 & 0.0 & 120 & 8510 \\
\hline $3 \mathrm{Si}+5 \mathrm{Ta}$ & 11.195 & 920 & 920 & solid & 0.0 & 0.0 & 80.9 & 906 \\
\hline $2 \mathrm{Si}+\mathrm{Th}$ & 6.659 & 2323 & 2323 & solid & 0.0 & 0.0 & 144 & 961 \\
\hline $2 \mathrm{Si}+\mathrm{Ti}$ & 3.134 & 1913 & $\geq 1773^{*}$ & $\mathrm{~s}-1$ & 0.0 & 0.0 & 308 & 967 \\
\hline $3 \mathrm{Si}+5 \mathrm{Ti}$ & 3.719 & 2548 & $\geq 2403^{*}$ & s-1 & 0.0 & 0.0 & 428 & 1590 \\
\hline $2 \mathrm{Si}+\mathrm{U}$ & 8.369 & 1663 & 1663 & solid & 0.0 & 0.0 & 106 & 940 \\
\hline $2 S i+V$ & 3.429 & 3341 & 2023 & $\mathrm{~s}-1$ & 0.0 & 0.0 & 700 & 2400 \\
\hline $2 \mathrm{Si}+\mathrm{W}$ & 7.480 & 1549 & 1549 & solid & 0.0 & 0.0 & 92.6 & 693 \\
\hline $\mathrm{Si}+\mathrm{Y}$ & 3.754 & 2108 & $*$ & $s-1$ & 0.0 & 0.0 & 275 & 1000 \\
\hline $\mathrm{Si}+2 \mathrm{Zr}$ & 5.291 & 2787 & $\geq 2198^{*}$ & liquid & 0.0 & 0.0 & 236 & 1250 \\
\hline $2 \mathrm{Si}+\mathrm{Zr}$ & 4.004 & 1988 & 1893 & liquid & 0.0 & 0.0 & 258 & 1040 \\
\hline $3 \mathrm{Si}+5 \mathrm{Zr}$ & 5.141 & 1132 & 1132 & solid & 0.0 & 0.0 & 255 & 1310 \\
\hline $2 U+17 Z n$ & 8.778 & 973 & 973 & solid & 0.0 & 0.0 & 60 & 530 \\
\hline $2 \mathrm{Zn}+\mathrm{Zr}$ & 6.816 & 1723 & $*$ & l-s & 0.0 & 0.0 & 170 & 1160 \\
\hline
\end{tabular}


Table 3 - Metal Combustion Reactions

\begin{tabular}{|c|c|c|c|c|c|c|c|c|}
\hline metal & oxide & $\begin{array}{c}\mathrm{MW}, \mathrm{g} / \mathrm{mol} \\
\text { (metal) }\end{array}$ & $\begin{array}{c}\rho_{\mathrm{TMD}}, \mathrm{g} / \mathrm{cm}^{3} \\
\text { (metal) }\end{array}$ & $\begin{array}{c}\Delta \mathrm{H}_{\mathrm{f}}^{\circ}, \\
\mathrm{kcal} / \mathrm{mol}\end{array}$ & $\begin{array}{l}\mathrm{Q}, \mathrm{cal} / \mathrm{g} \\
\text { of metal }\end{array}$ & $\begin{array}{c}\mathrm{Q}, \mathrm{cal} / \mathrm{cm}^{3} \\
\text { of metal }\end{array}$ & $\begin{array}{l}\mathrm{T}_{\text {fus }}, \mathrm{K}, \\
\text { of metal }\end{array}$ & $\begin{array}{l}\mathrm{T}_{\text {vap }}, \mathrm{K}, \\
\text { of metal }\end{array}$ \\
\hline $\mathrm{Ag}$ & $\mathrm{Ag}_{2} \mathrm{O}$ & 107.88 & 10.5 & 7.42 & 34.39 & 361.1 & 1234 & 2436 \\
\hline Al & $\mathrm{Al}_{2} \mathrm{O}_{3}$ & 26.98 & 2.70 & 400.5 & 7422 & 20040 & 933 & 2740 \\
\hline B & $\mathrm{B}_{2} \mathrm{O}_{3}$ & 10.82 & 2.5 & 304.0 & 14050 & 35120 & 2573 & 4139 \\
\hline $\mathrm{Be}$ & $\mathrm{BeO}$ & 9.01 & 1.85 & 143.2 & 15890 & 29400 & 1553 & 3243 \\
\hline $\mathrm{Bi}$ & $\mathrm{Bi}_{2} \mathrm{O}_{3}$ & 209.0 & 9.87 & 137.2 & 328.2 & 3240 & 544 & 1837 \\
\hline$\overline{\mathrm{Ce}}$ & $\mathrm{Ce}_{2} \mathrm{O}_{3}$ & 140.13 & 6.8 & 435.2 & 1553 & 10560 & 1048 & 3699 \\
\hline Co & $\mathrm{CoO}$ & 58.93 & 8.90 & 56.87 & 965.0 & 8589 & 1701 & 3201 \\
\hline $\mathrm{Cr}$ & $\mathrm{Cr}_{2} \mathrm{O}_{3}$ & 52.01 & 7.14 & 272.4 & 2619 & 18700 & 2180 & 2945 \\
\hline $\mathrm{Cs}$ & $\mathrm{Cs}_{2} \mathrm{O}$ & 132.91 & 1.90 & 75.95 & 571.4 & 1086 & 301 & 951 \\
\hline Cs & $\mathrm{Cs}_{2} \mathrm{O}_{3}$ & 132.91 & 1.90 & 111.3 & 837.3 & 1591 & 301 & 951 \\
\hline Cs & $\mathrm{CsO}_{2}$ & 131.91 & 1.90 & 62.04 & 466.8 & 886.9 & 301 & 951 \\
\hline $\mathrm{Cu}$ & $\mathrm{CuO}$ & 63.54 & 8.93 & 37.30 & 587.0 & 5244 & 1356 & 2843 \\
\hline $\mathrm{Cu}$ & $\mathrm{Cu}_{2} \mathrm{O}$ & 63.54 & 8.93 & 40.80 & 321.1 & 2868 & 1356 & 2843 \\
\hline $\mathrm{Fe}$ & $\mathrm{Fe}_{2} \mathrm{O}_{3}$ & 55.85 & 7.86 & 197.0 & 1764 & 13860 & 1811 & 3135 \\
\hline $\mathrm{Fe}$ & $\mathrm{Fe}_{3} \mathrm{O}_{4}$ & 55.85 & 7.86 & 267.3 & 1595 & 12540 & 1811 & 3135 \\
\hline $\mathrm{Hf}$ & $\mathrm{HfO}_{2}$ & 178.50 & 11.40 & 266.2 & 1491 & 17000 & 2495 & 4964 \\
\hline $\mathrm{La}$ & $\mathrm{La}_{2} \mathrm{O}_{3}$ & 138.92 & 6.15 & 428.7 & 1543 & 9489 & 1193 & 3730 \\
\hline $\mathrm{Li}$ & $\mathrm{Li}_{2} \mathrm{O}$ & 6.94 & 0.534 & 152.1 & 10960 & 5852 & 454 & 1620 \\
\hline $\mathrm{Mg}$ & $\mathrm{MgO}$ & 24.32 & 1.74 & 151.8 & 6241 & 10860 & 923 & 1363 \\
\hline $\mathrm{Mn}$ & $\mathrm{Mn}_{3} \mathrm{O}_{4}$ & 54.94 & 7.30 & 331.7 & 2012 & 14690 & .1519 & 2334 \\
\hline Mo & $\mathrm{MoO}_{3}$ & 95.95 & 9.01 & 178.1 & 1856 & 16720 & 2896 & 4952 \\
\hline $\mathrm{Nb}$ & $\mathrm{Nb}_{2} \mathrm{O}_{5}$ & 92.91 & 8.57 & 454.0 & 2443 & 20940 & 2750 & 5015 \\
\hline $\mathrm{Nd}$ & $\mathrm{Nd}_{2} \mathrm{O}_{3}$ & 144.27 & 7.01 & 433.6 & 1503 & 10530 & 1292 & 3341 \\
\hline $\mathrm{Ni}$ & $\mathrm{NiO}$ & 58.69 & 8.90 & 57.29 & 976.1 & 8688 & 1728 & 3187 \\
\hline $\mathrm{Pb}$ & $\mathrm{Pb}_{3} \mathrm{O}_{4}$ & 207.20 & 11.3 & 171.8 & 276.4 & 3133 & 601 & 2019 \\
\hline $\mathrm{Pd}$ & $\mathrm{PdO}$ & 106.40 & 12.0 & 27.60 & 259.4 & 3113 & 1828 & 3237 \\
\hline $\mathrm{Pt}$ & $\mathrm{Pt}_{3} \mathrm{O}_{4}$ & 195.09 & 21.4 & 64.05 & 109.4 & 2347 & 2042 & 4100 \\
\hline $\mathrm{Si}$ & $\mathrm{SiO}_{2}$ & 28.09 & 2.49 & 217.7 & 7750 & 19298 & 1687 & 2628 \\
\hline $\mathrm{Sn}$ & $\mathrm{SnO}_{2}$ & 118.70 & 7.31 & 138.8 & 1169 & 8548 & 505 & 2876 \\
\hline $\mathrm{Sr}$ & $\mathrm{SrO}_{2}$ & 87.62 & 2.6 & 153.3 & 1750 & 4549 & 1042 & 1657 \\
\hline $\mathrm{Ta}$ & $\mathrm{Ta}_{2} \mathrm{O}_{5}$ & 180.95 & 16.6 & 489.0 & 1351 & 22430 & 3290 & 5698 \\
\hline $\mathrm{Th}$ & $\mathrm{ThO}_{2}$ & 232.04 & 11.2 & 293.5 & 1265 & 14170 & 2023 & 5063 \\
\hline $\mathrm{Ti}$ & $\mathrm{TiO}_{2}$ & 47.90 & 4.50 & 225.8 & 4714 & 21210 & 1693 & 3560 \\
\hline $\mathrm{U}$ & $\mathrm{U}_{3} \mathrm{O}_{8}$ & 238.07 & 18.9 & 854.4 & 1196 & 22610 & 1408 & 4091 \\
\hline $\mathrm{V}$ & $\mathrm{V}_{2} \mathrm{O}_{5}$ & 50.95 & 5.87 & 370.6 & 3634 & 21350 & 2183 & 3653 \\
\hline W & $\mathrm{WO}_{2}$ & 183.86 & 19.3 & 140.9 & 766.6 & 14790 & 3695 & 5936 \\
\hline $\mathrm{W}$ & $\mathrm{WO}_{3}$ & 183.86 & 19.3 & 201.5 & 1096 & 21150 & 3695 & 5936 \\
\hline$Y$ & $\mathrm{Y}_{2} \mathrm{O}_{3}$ & 88.92 & 4.47 & 501.4 & 2819 & 12600 & 1799 & 3611 \\
\hline $\mathrm{Zn}$ & $\mathrm{ZnO}$ & 65.38 & 7.14 & 83.76 & 1281 & 9147 & 692 & 1180 \\
\hline $\mathrm{Zr}$ & $\mathrm{ZrO}_{2}$ & 123.22 & 5.68 & 263.0 & 2135 & 12130 & 2125 & 4650 \\
\hline
\end{tabular}

